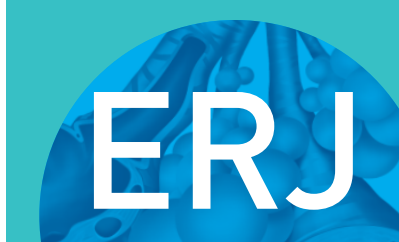

open research

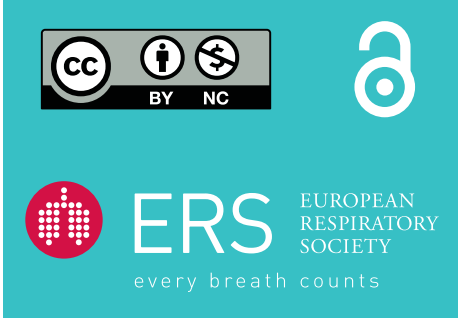

\section{High-dose glucocorticoid treatment of near-fatal bocavirus lung infection results in rapid recovery}

\author{
To the Editor:
}

Human bocavirus (HBoV), which belongs to Parvoviridae, is a well-defined pathogen of respiratory infections, particularly in young children [1]. In contrast, the frequency of $\mathrm{HBoV}$ infections in adults with respiratory symptoms is virtually unknown and its causative role in respiratory failure is debated $[1,2]$. Furthermore, the observation that dexamethasone is beneficial in COVID-19 patients with respiratory failure requiring respiratory support has gained great interest [3]. However, whether glucocorticoid treatment is useful in other severe viral respiratory diseases is a matter of controversy [4].

Here, we report on a 58-year-old, obese (body mass index $35 \mathrm{~kg} \cdot \mathrm{m}^{-2}$, Eastern Cooperative Oncology Group performance status 1), Caucasian male who presented at Charité University Hospital (Berlin, Germany) on 25 February 2020 with a 6-day history of fever and shortness of breath. 3 months earlier, he had received BEAM (carmustine, etoposide, cytarabine and melphalan) high-dose chemotherapy and autologous stem cell transplantation (autoSCT) for relapsed Hodgkin's lymphoma, resulting in complete remission. Relevant medical history included psoriasis, diabetes mellitus type 2, permanent atrial fibrillation and COPD of Global Initiative for Chronic Obstructive Lung Disease stage II, with no relevant previously documented pulmonary structural alterations. Concomitant medication on admission consisted of acyclovir, pantoprazole, digitoxin, bisoprolol, sitagliptin, metformin, insulin and acitretin, all not considered as causative for the clinical symptoms. On admission, peripheral oxygen saturation demonstrated hypoxaemia $\left(S_{\mathrm{pO}_{2}} 88 \%\right.$ ), C-reactive protein (CRP) level was $50.9 \mathrm{mg} \cdot \mathrm{L}^{-1}$ (normal range $\left.<5 \mathrm{mg} \cdot \mathrm{L}^{-1}\right)$, and chest radiography disclosed diffuse reticular, interstitial infiltrations of the lung. As the patient deteriorated, accompanied by further CRP increase to $92.9 \mathrm{mg} \cdot \mathrm{L}^{-1}$, during a 7 -day course $(25$ February to $3 \mathrm{March}$ ) of antibiotic treatment with piperacillin/tazobactam and ciprofloxacin, a subsequent lung computed tomography $(\mathrm{CT})$ scan (figure 1a) revealed bilateral pulmonary infiltrates with interstitial pattern, ground-glass opacities, (pre-existing) bullae and consolidation in the upper-left lobe. Small-volume bronchoalveolar lavage (BAL) and lung biopsy were performed. On 5 March, the patient finally required mechanical ventilation due to respiratory failure without signs of overt sepsis, with an initial positive end-expiratory pressure of $5 \mathrm{cmH}_{2} \mathrm{O}$; arterial oxygen tension/inspiratory oxygen fraction ratio was $136 \mathrm{mmHg}$, suggesting moderate acute respiratory distress syndrome (ARDS). Extended examinations of the BAL for infectious pathogens (bacterial culture and microscopy; and PCR-based respiratory panel analyses including various infectious agents (viruses (adenovirus, $\mathrm{HBoV}$, cytomegalovirus, coronavirus including severe acute respiratory syndrome (SARS) coronavirus 2, Epstein-Barr virus (EBV), entero/rhinovirus, human herpesvirus 6, herpes simplex virus (HSV)-1 and HSV-2, influenzavirus A/B, metapneumovirus, parainfluenzavirus 1-4, respiratory syncytial virus $A / B$, varicella zoster virus), bacteria (Bordetella spp., Chlamydia spp., Legionella spp. and Mycoplasma spp.) and Pneumocystis jirovecii)) revealed positivity for $\mathrm{HBoV}$ (and low-level detection of EBV (2390 copies (cp) per $\mathrm{mL}$ ) in BAL but not in blood, judged not to be clinically relevant) but no other pathogens.

We considered $\mathrm{HBoV}$ infection followed by excessive immune response, possibly aggravated by immune reconstitution after autoSCT, as underlying pathogenic events. A high concentration of $\mathrm{HBoV}$ genome

@ERSpublications

Human bocavirus ( $\mathrm{HBoV}$ ) has to be considered a life-threatening pathogen in adults with atypical pneumonia. Pulsed high-dose glucocorticoid treatment may be beneficial in patients suffering from severe pulmonary disease caused by $\mathrm{HBoV}$ or other viruses. https://bit.ly/3epiMyO

Cite this article as: Obenaus $\mathrm{M}$, Schildgen $\mathrm{O}$, Schürmann $\mathrm{D}$, et al. High-dose glucocorticoid treatment of near-fatal bocavirus lung infection results in rapid recovery. ERJ Open Res 2021; 7: 00060-2021 [https://doi.org/10.1183/23120541.00060-2021].

Copyright $\odot$ The authors 2021. This version is distributed under the terms of the Creative Commons Attribution NonCommercial Licence 4.0. For commercial reproduction rights and permissions contact permissions@ersnet.org 

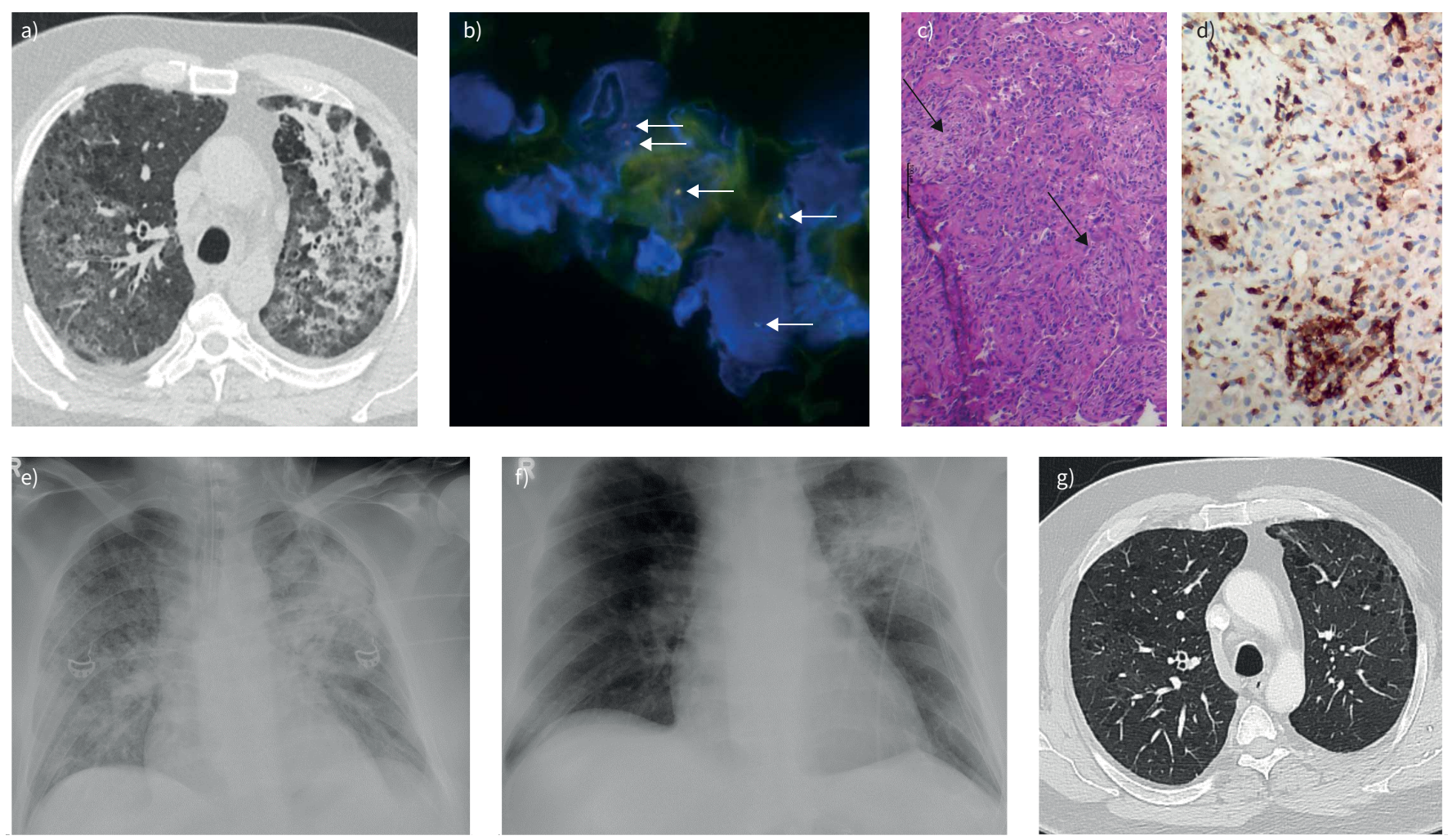

FIGURE 1 Radiomorphological and pathological-anatomical findings of a patient with near-fatal human bocavirus (HBoV) infection. a) Computed tomography (CT) image after a 7-day course of antibiotic therapy shows bilateral pulmonary infiltrates with interstitial pattern and ground-glass opacities (3 March 2020). b) HBoV-specific fluorescence in situ hybridisation in lung biopsy. Head and tail HBoV genome regions were detected with specific fluorescence probes and tissue was counterstained with DAPI. Yellow and orange dots (arrows) indicate codetection of the head and tail regions of HBoV-DNA. c) Lung biopsy showing fibroblast proliferation (arrows) (haematoxylin and eosin staining). d) Strong infiltration with T-lymphocytes (CD3 staining in brown) in the lung tissue reflecting excessive immune response. e) Chest radiography (anteroposterior view) at initiation of glucocorticoid treatment (day 1) showing multifocal opacities in both lungs with foci of consolidation in the upper-left lobe (5 March 2020), and f) at day 4, demonstrating resolution of the multifocal opacities with demarked consolidation in the upper left lobe (8 March 2020). g) CT image 2 months after treatment demonstrates complete resolution of infiltrates with remaining pre-existing small bullae (26 May 2020 ).

copies $\left(3.2 \times 10^{8} \mathrm{cp} \cdot \mathrm{mL}^{-1}\right.$ in $\left.\mathrm{BAL}\right), \mathrm{HBoV}$ detection by fluorescence in situ hybridisation (figure $1 \mathrm{~b}$ ) and pronounced T-lymphocytic infiltrates (figure $1 \mathrm{c}$ and $\mathrm{d}$ ) in the lung tissue confirmed this scenario. Given the lack of $\mathrm{HBoV}$-specific treatments, we aimed to mitigate the immune reaction and subsequent pulmonary damage by pulsed high-dose glucocorticoid therapy (5 March (day 1), $500 \mathrm{mg}$ prednisolone; days 2 and 3, $1000 \mathrm{mg}$ methylprednisolone) followed by rapid reduction to $20 \mathrm{mg} \cdot \mathrm{day}^{-1}$ prednisolone and tapering. Respiratory parameters and radiological findings improved rapidly (figure 1e and f), allowing termination of mechanical ventilation on day 4 ( 8 March) of intensive care unit treatment and discharge on 17 March. In parallel to the clinical improvement, CRP values rapidly decreased and remained normal. A CT scan 2 months after treatment confirmed complete resolution of all infiltrates (figure $1 \mathrm{~g}$ ).

Given the virtually unknown frequency of $\mathrm{HBoV}$ in adult patients with respiratory symptoms [1], we retrospectively analysed a total of 5328 consecutive adult respiratory samples, mainly consisting of tracheobronchial secretions and BALs, and which were all examined by PCR-based respiratory panel analyses, for $\mathrm{HBoV}$. We identified $17 \mathrm{HBoV}$-positive patients, most of them heavily immunocompromised. Given the reconstitution of white blood cells $\left(4.45 \mathrm{~nL}^{-1}\right.$, normal range $\left.3.90-10.50 \mathrm{~nL}^{-1}\right)$ with normal neutrophils $\left(3.03 \mathrm{~nL}^{-1}\right.$, normal range $\left.1.50-7.70 \mathrm{~nL}^{-1}\right)$ and slightly reduced lymphocytes $\left(0.95 \mathrm{~nL}^{-1}\right.$, normal range $\left.1.10-4.50 \mathrm{~nL}^{-1}\right)$, even though platelets $\left(30 \mathrm{~nL}^{-1}\right.$, normal range $\left.150-370 \mathrm{~nL}^{-1}\right)$ and haemoglobin $\left(8.9 \mathrm{mg} \cdot \mathrm{dL}^{-1}\right.$, normal range $\left.13.5-17.0 \mathrm{mg} \cdot \mathrm{dL}^{-1}\right)$ were reduced on admission, as well as almost normal immunoglobulin levels (IgG $6.61 \mathrm{~g} \cdot \mathrm{L}^{-1}$, normal range $7.00-16.00 \mathrm{~g} \cdot \mathrm{L}^{-1}$; $\operatorname{IgA} 1.72 \mathrm{~g} \cdot \mathrm{L}^{-1}$, normal range $0.7-4.00 \mathrm{~g} \cdot \mathrm{L}^{-1}$; and $\operatorname{IgM} 0.30 \mathrm{~g} \cdot \mathrm{L}^{-1}$, normal range $0.40-2.30 \mathrm{~g} \cdot \mathrm{L}^{-1}$ ) at the time of $\mathrm{HBoV}$-induced respiratory failure, we judged our patient to be largely immunocompetent. Thus, even though rare, $\mathrm{HBoV}$ must be considered a life-threatening pathogen in adults with atypical pneumonia and should be included in the respective diagnostic work-up, with $\mathrm{HBoV}-\mathrm{DNA}$ quantification if positive. 
Only recently, it has been demonstrated that dexamethasone is beneficial in COVID-19 patients with respiratory failure requiring respiratory support [3]; however, the benefit of glucocorticoid treatment in other severe viral respiratory diseases is unclear [4]. In contrast to the dexamethasone scheme used in the RECOVERY trial in COVID-19 patients (6 mg once daily for up to 10 days), we applied a pulsed high-dose glucocorticoid therapy scheme deduced from treatment of autoimmune diseases. Notably, the application of such pulsed "very high-dose" glucocorticoid therapy is supposed to extend the glucocorticoid mode of action compared to lower doses $[5,6]$. We assume that this short-term high-dose glucocorticoid therapy effectively dampened the detrimental virus-induced immune response, most likely by glucocorticoid-induced cell death of immune effector cells including infiltrating T-lymphocytes, and anti-inflammatory effects by modulation of monocyte and macrophage function [7, 8]. Furthermore, glucocorticoid therapy can inhibit fibroblast proliferation and support the clearance of inflammation-induced tissue damage [7]. We are aware of glucocorticoid therapy-induced side-effects; that corticosteroid treatment of ARDS or SARS patients, or patients suffering from viral pneumonia, has generated conflicting results [4, 5, 9-11]; and that prolonged intake of corticosteroids predisposes to invasive aspergillosis in the context of severe influenza infection [12]. However, short pulsed high-dose glucocorticoid therapy has been used for decades for the treatment of various severe autoimmune diseases, with limited and well-known side-effects [13]. Therefore, we decided to use such a "very high-dose" short-term scheme and not a lower-dose, longer-lasting scheme for our patient. We believe that in selected patients, in which the virus-induced immune response is profound and likely to be more harmful to lung tissue than damage by the virus itself, and in which fatal lung damage has not yet passed the point of no return, glucocorticoid therapy may preserve lung tissue and function. Notably, most recently, it has been shown that severe COVID-19 patients benefit from pulsed methylprednisolone administration if given during the early phase of pulmonary infection [14]. Thus, high-dose glucocorticoid therapy may be beneficial in patients suffering from severe pulmonary disease caused by $\mathrm{HBoV}$, as demonstrated in our case, or other viruses.

Matthias Obenaus $\oplus^{1}$, Oliver Schildgen ${ }^{2}$, Dirk Schürmann ${ }^{3}$, Ann-Christin von Brünneck ${ }^{4}$, Martin Janz ${ }^{1,5}$, Ulrich Keller ${ }^{1,5}$, Bernhard Gebauer ${ }^{6}$, Johannes Schilling ${ }^{7}$, Stefan Schwartz $\oplus^{1}$, Benedikt Weissbrich ${ }^{8}$, Thomas Schneider ${ }^{9}$, Jörg Hofmann ${ }^{10}$ and Stephan Mathas ${ }^{1,5}$

${ }^{1}$ Hematology, Oncology and Tumor Immunology, Charité - Universitätsmedizin Berlin, corporate member of Freie Universität Berlin and Humboldt-Universität zu Berlin, Berlin, Germany. ${ }^{2}$ Institute of Pathology, Kliniken der Stadt Köln gGmbH, Kliniken der Privaten Universität Witten/Herdecke mit Sitz in Köln, Cologne, Germany. ${ }^{3}$ Infectious Diseases and Respiratory Medicine, Charité - Universitätsmedizin Berlin, Berlin, Germany. ${ }^{4}$ Pathology, Charité - Universitätsmedizin Berlin, Berlin, Germany. ${ }^{5}$ Experimental and Clinical Research Center, a cooperation between the Charité and the Max-Delbrück-Center for Molecular Medicine, Berlin, Germany. ${ }^{6}$ Radiology, Charité - Universitätsmedizin Berlin, Berlin, Germany. ${ }^{7}$ Nephrology and Medical Intensive Care, Charité - Universitätsmedizin Berlin, Berlin, Germany. ${ }^{8}$ Institute for Virology and Immunbiology, University Würzburg, Würzburg, Germany. ${ }^{9}$ Gastroenterology, Infectious Diseases and Rheumatology, Charité - Universitätsmedizin Berlin, Berlin, Germany. ${ }^{10}$ Institute of Virology, Charité - Universitätsmedizin Berlin, Berlin, Germany.

Correspondence: Stephan Mathas, Hematology, Oncology and Tumor Immunology and Max-DelbrückCenter for Molecular Medicine, Charité - Universitätsmedizin Berlin, Hindenburgdamm 30, D-12200 Berlin, Germany. E-mail: stephan.mathas@charite.de

Received: 12 Feb 2021 | Accepted: 27 Feb 2021

Conflict of interest: M. Obenaus reports personal fees from Novartis during the conduct of this study. O. Schildgen has nothing to disclose. D. Schürmann has nothing to disclose. A-C. von Brünneck has nothing to disclose. M. Janz has nothing to disclose. U. Keller reports personal fees from Takeda, Hexal, Abbvie, Pentizapharm and AstraZeneca, and personal fees and other support from Roche, Janssen-Cilag, Pfizer and BMS, outside the submitted work. B. Gebauer reports personal fees from Parexek/CALYX, ICON, Bayer, Siemens, Roche, Merck, IPSEN, Pfizer, Elsai, MSD, Pharmcept, Guerbet, Ewimed and Sirtex Medicalm outside the submitted work. J. Schilling has nothing to disclose. S. Schwartz reports personal fees from Pfizer, BTG Intl Inc., MSD and Novartis, personal fees and nonfinancial support from Gilead Sciences, AMGEN and Jazz Pharmaceuticals, and nonfinancial support from Basilea, outside the submitted work. B. Weissbrich has nothing to disclose. T. Schneider has nothing to disclose. J. Hofmann has nothing to disclose. S. Mathas has nothing to disclose.

\section{References}

$1 \quad$ Qiu J, Söderlund-Venermo M, Young NS. Human parvoviruses. Clin Microbiol Rev 2017; 30: 43-113.

2 Dieninghoff D, Karagiannidis C, Straßmann S, et al. Fatal HBoV-1 infection in adult female cystic fibrosis patient. Hum Pathol Case Rep 2017; 7: 51-52. 

693-704.

4 Ruuskanen O, Lahti E, Jennings LC, et al. Viral pneumonia. Lancet 2011; 377: 1264-1275.

5 Stahn C, Buttgereit F. Genomic and nongenomic effects of glucocorticoids. Nat Clin Pract Rheumatol 2008; 4: 525-533.

6 Buttgereit F, da Silva JAP, Boers M, et al. Standardized nomenclature for glucocorticoid dosages and glucocorticoid treatment regimens: Current questions and tentative answers in rheumatology. Ann Rheum Dis 2002; 61: 718-722.

7 Cain DW, Cidlowski JA. Immune regulation by glucocorticoids. Nat Rev Immunol 2017; 17: $233-247$.

8 Ehrchen JM, Roth J, Barczyk-Kahlert K. More than suppression: glucocorticoid action on monocytes and macrophages. Front Immunol 2019; 10: 2028.

9 Steinberg KP, Hudson LD, Goodman RB, et al. Efficacy and safety of corticosteroids for persistent acute respiratory distress syndrome. $N$ Engl J Med 2006; 354: 1671-1684.

10 Villar J, Ferrando C, Martínez D, et al. Dexamethasone treatment for the acute respiratory distress syndrome: a multicentre, randomised controlled trial. Lancet Respir Med 2020; 8: 267-276.

11 Sung JJY, Wu A, Joynt GM, et al. Severe acute respiratory syndrome: report of treatment and outcome after a major outbreak. Thorax 2004; 59: 414-420.

12 Schauwvlieghe AFAD, Rijnders BJA, Philips N, et al. Invasive aspergillosis in patients admitted to the intensive care unit with severe influenza: a retrospective cohort study. Lancet Respir Med 2018; 6: 782-792.

13 Weusten BLAM, Jacobs JWG, Bijlsma JWJ. Corticosteroid pulse therapy in active rheumatoid arthritis. Semin Arthritis Rheum 1993; 23: 183-192.

14 Edalatifard M, Akhtari M, Salehi M, et al. Intravenous methylprednisolone pulse as a treatment for hospitalised severe COVID-19 patients: Results from a randomised controlled clinical trial. Eur Respir J 2020; 56: 2002808. 